\title{
IMPLEMENTATION OF CELL PHONE DETECTION MOBILE ROBOT FOR RESTRICTED AREAS USING NODEMCU
}

\author{
Hamzah M. Marhoon', Zeyad A. Karam², Aymen M. Al-Kadhimi ${ }^{3}$ \\ ${ }^{1}$ Department of Computer Techniques Engineering, Al-Esraa University College, Iraq \\ ${ }^{2,3}$ College of Information Engineering, Al-Nahrain University, Iraq \\ hamza@esraa.edu.iq ${ }^{1}$,zyad.karam@coie-nahrain.edu.iq ${ }^{2}$, aymen.mohammed@coie-nahrain.edu.iq ${ }^{3}$
}

(Received: 16/01/2018; Accepted: 25/02/2018)

\begin{abstract}
This paper involves the design and implementation of cell phone detection mobile robot. This is applicable in examination halls, private conferences and meeting rooms in which the use of cell phones is highly restricted. The robot is able to detect the presence of unauthorized communications by active phones and then distort them. The detection process is achieved by implementing an electrical circuit for sensing undesirable signals and using NodeMCU for interfacing the robot with a main computer unit. The main computer unit is used as a controlling platform in terms of mobile robot navigation as well as detection and jamming activating. This is accomplished by creating a graphical control panel programmed using a special HTML script. In this work, the cell phone detection mobile robot has been applied in an examination hall to simulate real educational environment. The robot has detected active calls by cell phones with a circle diameter of 1.2 meter and then jammed them directly. The whole area has been covered for detection and jamming by roaming the robot wirelessly via the remote main computer. Different voltage measurements for different detection distances have been recorded.
\end{abstract}

Keywords—mobile robot; cell phone; detection circuit; NodeMCU.

\section{INTRODUCTION}

The development of robotics and communication takes a large scale in the recent years. The use of mobile robot in communication filed gives a strong motivation in this context, especially the idea of controlling and driving subjected to the communication and interfacing protocols. That enables implementing different applications realized with the mobile robot [1].

One of these interesting applications is utilizing mobile robot as detecting and cutting mobile unit's signal, and that robot can be navigated and driven via a main computer unit through some communication protocols. The purpose of using this type of application is to prevent the misuse of mobile cell phones in some special educational environments such as college premises and examination halls. The traditional manual inspection of mobile handsets does not necessarily guarantee a full detection, in other words, some students may pass through with their undetected phones or smart devices, which may violate university's regulations. Those handheld smart appliances might be inappropriately exploited in exams by students who are being connected to other intruders sitting outside the examination halls for illegal answers sharing proposes. That unethical behavior is clearly declared as cheating and should be highly taken into considerations. Even though it is true that the detection of such malpractices is the invigilators responsibility, it is essential to apply modern technologies in such scenarios to eliminate those possible vulnerabilities.

This paper focuses on testing mobile robot detection unit in examination halls. The detection is done using NodeMCU instilled in mobile robot which has the ability to detect cellular signals by interfacing with a detection circuit to a main computer. This unit monitored by colleges and once the mobile robot unit detects a cell phone signal, it directly fires an alarm to notify invigilators that there is an unauthorized cell phone being exploited by a student. A 
special designed hypertext markup language (HTML) script used for driving the mobile robot and controlling the jammer. The driving signal of mobile robot is controlled manually via the main computer unit and passing through the NodeMCU. Also, to easily control the mobile robot via the main computer, a wireless camera is instilled in front of the robot's body. This camera is planted to monitor the target examination hall and help driving the mobile robot roaming among students till a detection of an unfavorable signal is recorded. Subsequently, the mobile robot fires a cutting signal through activating the jammer Yt_003 for a short period of time to ensure jamming that unauthorized signal. The detector circuit is designed using one stage operational amplifier for detecting the radio frequency (RF) signals operating at the Universal Mobile Telecommunications Service (UMTS) bands.

This paper presents this project by offering: (1) design and implementation of mobile cell phone detection circuit; (2) interface between mobile detector circuit with a main computer unit using NodeMCU; (3) design of a special HTML script for jammer switching and mobile robot navigation; (4) implementation of an interfacing circuit between the NodeMCU and mobile robot motors drivers.

\section{LITERATURE REVIEW}

In the literature, a great applying compatibility between communication systems and mobile robots has been achieved so far. Recently, many research works have been done with cell phone detection, some with valued contributions and others with considerable limitations. Dar in 2014 [2] designed a system that dictated the presence of GSM signals of an unauthorized user in restricted areas. That system could trigger another device to restrict users from getting services. In addition, jamming GSM frequency signal upon detection is offered by the system to prevent any unauthorized connections. Another cell phone detector circuit was presented by Nyamawe and Mtonyole [3] in which Arduino micro controller was used in the implementation. The Arduino detection circuit used for replacing the existing approaches to ensure that students do not hold mobile phones in exams instead of doing that inspection manually during entrance. These preceding research papers were followed by a study proposed in 2015 [4] by Deshpande and Jadhav. In their research, they suggested an intelligent mobile phone detection project to block any illegal active mobile (GSM) communications. That project could be applicable to private meetings, examination halls, defense establishments, military camps and hospitals.

In the subsequent year, 2016, three research works were considered in this context. The first one proposed by Kumar [5] who used mobile phone that works remotely with mobile robot. The control technology of the used robot was done by three stages: perception, processing and action (actuators). Generally, the preceptors were sensors instilled in the robot, processing was done by microcontrollers and the work was performed using motors. The second study was presented in [6] in which an RF signals detection circuit was implemented. Gayathri and Sivasakthi applied that design to detect both audio and video transmission signals from cameras and microphones with cutting the incoming and outgoing calls from mobile phone. The RF signals detector was performing its task by indicating a beep alarm with an LCD to display the indication and inform the admin by a code programmed in microcontroller. On the other hand, two detection circuits were proposed and tested with executing system evaluation were outlined by Ataro et al [7], and that was the third study to be reviewed in 2016. The designed circuit particularly focused on cutting the RF (global system for mobile communications GSM) cell phone signal from accessing users in the pre-defined areas. 
In the most recent year, 2017, Ambulge et al. [8] proposed a wireless motion of mobile robot using Bluetooth module to control robot's movements via a cell phone. That cell phone has Android based application and a Bluetooth signal for controlling the mobile robot's movements wirelessly. The Bluetooth module was interfaced with Arduino card to take the required actions from the cell phone and execute them on the mobile robot by using two DC motors. The robot movement actions were forward, reverse, left, right and stop. In addition, a metal detector circuit that can sense metals instilled in the robot's path with sending alarming signals in case of addressing a detection. Another study in the same year presented by Parvateesam and Kumar [9] who designed a circuit that can detect cell phone signals at transmission term and sends indication to the alarm that being fired continuously until the RF signal transmission interrupted. That proposed system used for detecting calls, SMS and video communications even when the cell phone was in the silent mode.

In terms of the limitations that the preceding studies may suffer from, there are two main points could be outlined. Firstly, the research tended to focus on installing the detection circuit on a constant specific area so that it did not cover the whole target place. Secondly, there was no software used to drive the mobile robot unit through long distances because of using Bluetooth module.

According to those pre-mentioned limitations, this paper will manage and introduce solutions to increase efficiency of the cell phone detection mobile robot by:

- Using a modified design of cell phone detector circuit instilled in mobile robot.

- Sending indication signal by the detector to fire an alarm and then to enable the main computer user running the jammer Yt_003 for a period of time to cut any unauthorized cell phone connections.

- Transmitting a video monitoring signal wirelessly to the main computer using a camera instilled in front of mobile robot.

- Creating a graphical user interface (GUI) control panel by programming a special HTML script used to enable the user controlling and navigating the mobile robot in the target zones with switching the jammer on and off.

- Receiving driving signals by the NodeMCU from the main computer wirelessly and then forwarding them to the DC motors drivers.

\section{SYSTEM DESIGN}

The main system components in terms of hardware are each of the detection circuit, NodeMCU, mobile robot unit, jammer and wireless camera in addition to a GUI panel set using HTML script as software. Firstly, any unauthorized RF signals is discovered by the detection circuit and then a buzzer fires on to notify invigilators about the undesired connections. Afterwards, a notification, as a text, is sent to the main computer user so that they control the jammer Yt_003 to turn it on for a period of time to ensure that the detected signal is blocked. The main computer user, via the GUI panel, can control the jammer and drive the mobile robot motors to the desired path. Those driving signals are received wirelessly via the NodeMCU to be sent then to the motors. The mobile robot unit is utilized to ensure covering the whole restricted area by handling each of the mobile detection unit and the signal cutting unit while roaming around as well as benefiting an instilled wireless camera to ease driving. That would enable the user smoothly driving 
the mobile robot to the required areas or paths. The overall block diagram for the mobile detection system shown in Fig. 1. Next, the system parts will be explained individually in terms of design and structure.

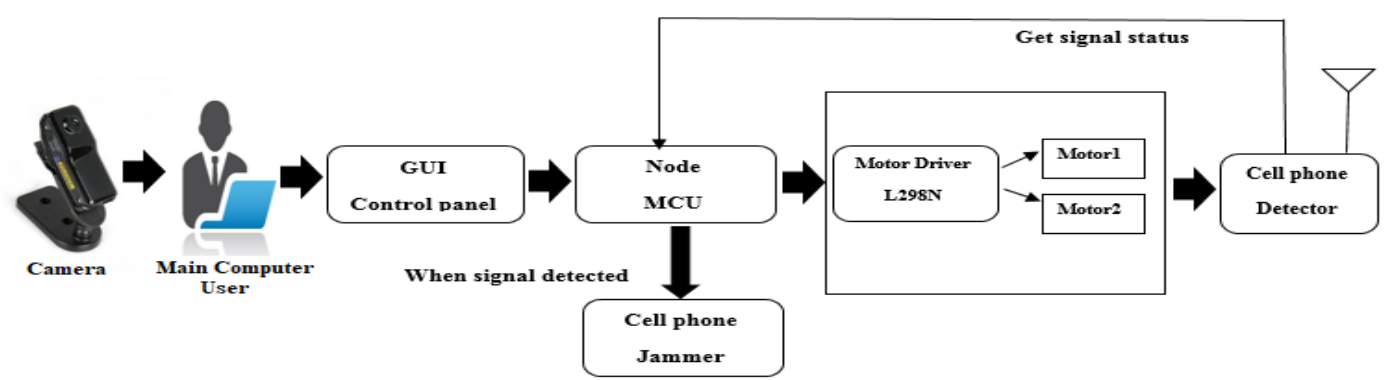

Figure 1. The overall block diagram of the mobile detection unit

\section{A. Cell Phone Detection Circuit}

The first element of the system is the cell phone detection circuit in which the operational amplifier LM358AD is used as an RF signals detector ranged from low frequencies up to UMTS bands. This detection is sensed by antenna connected to the LM358AD as inverted input, and the other input used to calibrate the circuit for optimal detection with different wave lengths. That optimal detection has been achieved with a maximum detection range by choosing the optimal resistance (R3) value to be $220 \mathrm{~K} \Omega$ without the need for resetting the sensing part of LM358AD. Electromagnetic RF radiation was sensed via detection circuit, when an active phone call is addressed, by the capacitor $\mathrm{C} 1$ which is working as a detector for this RF signal. Once the $\mathrm{C} 1$ ingests the RF signal and passing it to the LM358AD inputs to be reflected as light emitting diode LED flashing. After that, the output signal of LM358AD being inputted to the transistor BC548BP which works as a switch for buzzer and LED. Fig. 2 shows the schematic design of the improved cell phone detection circuit using Multisim electronic simulator.

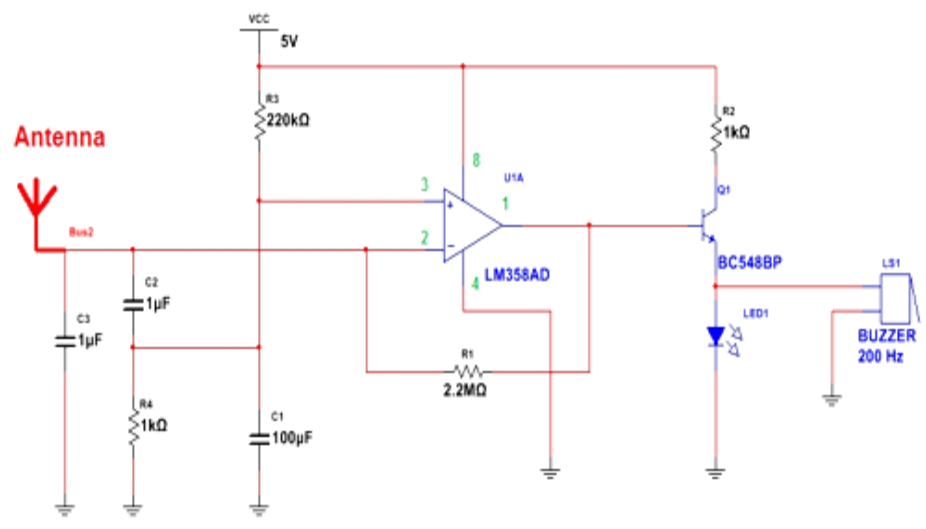

Figure 2. Schematic circuit of the cell phone detector

This RF circuit can circularly detect the presence of active cell phones calls within a diameter of 1.2 meter, therefore, it is perfectly applicable in meeting rooms, private conferences and examination halls in which the using of cell phones 
is forbidden. Furthermore, the detection circuit can spot the incoming and the outgoing voice calls, video transmission and short text massages in all mobile modes even so in silent. When RF signals are detected due to activated cell phone, the circuit LED and buzzer start illuminating and ringing respectively at the same time in a continues mode until the signals are cut by jammer. The cell phone detection circuit implemented on Veroboard is depicted in Fig. 3.

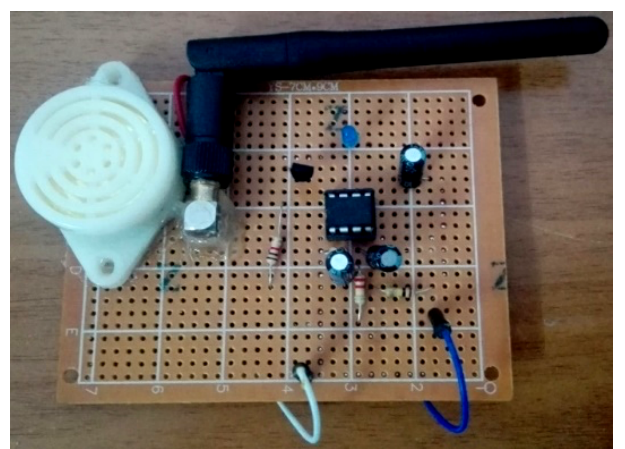

Figure 3. Implemented cell phone detection circuit on Veroboard

\section{B. Node $\mathrm{MCU}(\mathrm{Sub})$}

The NodeMCU with integrated ESP8266is Wi-Fi SoC developed by Espressif Systems. The ESP8266 designed to solve the Wi-Fi networking problem by acting as a bridge between embedded microcontroller to the Wi-Fi module. NodeMCU microcontroller also has ability of running applications loaded to it. The NodeMCU can use USB micro cable; the NodeMCU development kit can be connected to laptops and run it like Arduino [10]. The NodeMCU embedded system is shown in Fig. 4.

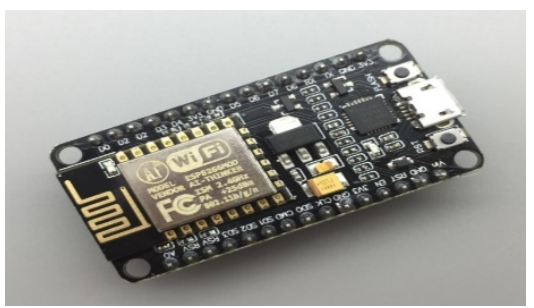

Figure 4. NodeMCU module

The second attribute of this work is the use of NodeMCU for wirelessly connecting the mobile robot with main computer. That process includes two stages.

Firstly, receiving the manual control signals of jammer from the HTML script and interfacing it by using a relay module as shown in Fig. 5. The jammer working principle is blocking signals in the network by sending a signal with huge power "J" and with a frequency similar to that signal to be jammed 'M". In consequence, the signal "J" by the jammer causes interfering and then blocking the signal " $\mathrm{M}$ " by unauthorized user. The jammer distorts the frequency signals between base stations and mobile handsets in two modes: uplink and downlink. On the other hand, humidity, temperature, presence of obstacles and buildings are factors may affect the jammer's accuracy. The operation principle of the jammer is shown as a block diagram in Fig. 6. 


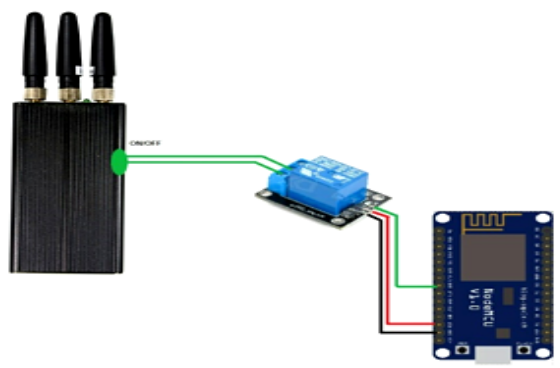

Figure 5. Interfacing of NodeMCU with jammer module

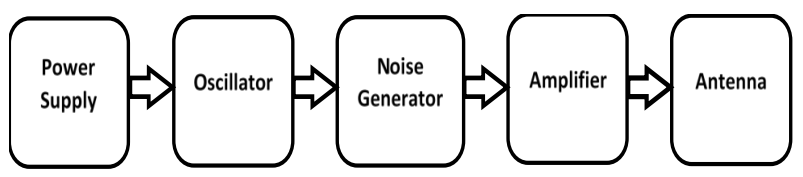

Figure 6. Block diagram of jammer operation

Secondly, receiving the manual control signals from the main computer wirelessly via Wi-Fi antenna and then sending them to the motor drivers (L293n). These signals are being sent as digital output from the NodeMCU embedded system. Fig. 7 shows the interfacing circuit of the robot motor drivers with the NodeMCU.

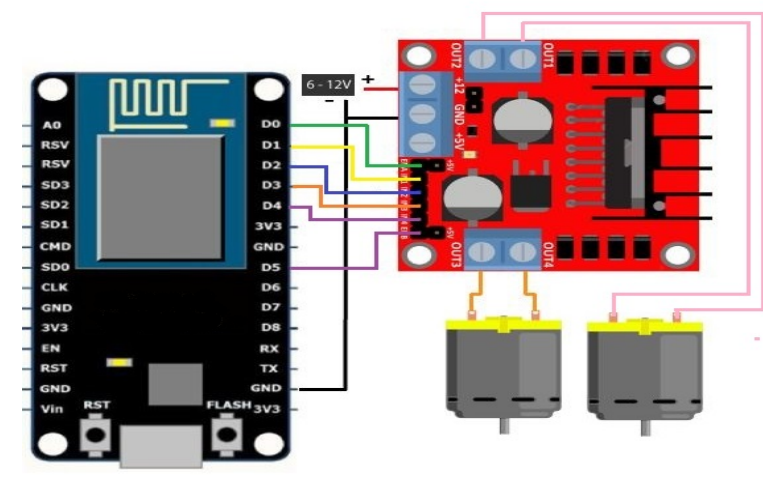

Figure 7. Interfacing of NodeMCU with L293n motor driver

\section{HTML Script for GUI Panel}

The third attribute of this work is programming a GUI window using an HTML script. That script is required to enable users controlling and navigating the mobile robot with switching jammer on and off via GUI.

The HTML script is formatted to desired GUI buttons by programing the NodeMCU using Arduino integrated development environment (IDE) language. That is done by importing "Esp8226wifi.h" library to enable user from writing the HTML and IDE codes at the same time. At first, the generation of local IP address is required, and that is assigned by identifying the local Wi-Fi "ssid" and "password" to the IDE to be connected to Wi-Fi using the command "WiFi.begin (ssid, password)". Also, the baud rate for serial communication with Wi-Fi is set to 115200 bit/sec. Then, the local IP address will be generated according to the local Wi-Fi signal by the command "Serial.print (WiFi.localIP());", and this local IP is varying according to the physical address from computer to another. After that, 
the generated IP is taken to browser to access the GUI in which the control buttons are placed. Those buttons include the robot driving buttons: "Forward", " Backward", "Left" and "Right" as well as the jammer control commands: "Turn on" and "Turn off". Fig. 8 depicts the Wi-Fi connection and generated local IP address window and the GUI control panel designed in HTML.

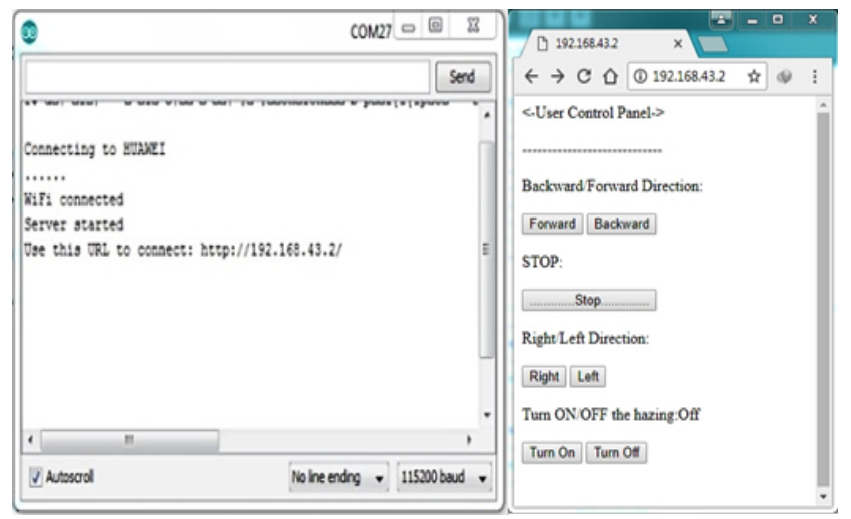

Figure 8. GUI control panel of the mobile robot.

\section{MOBILE ROBOT UNIT}

The purpose of using the mobile robot in this system is to cover the whole defined areas where unauthorized cell phone communications are intended to be jammed. That zone coverage is achieved since the mobile robot has the ability to roam over different paths. This motion is executed by the main computer user who is manually driving the mobile robot after getting the vision angles using a wireless camera. The mobile robot structure includes the main frame sheet which stands on three wheels, two of them guided by DC motors for navigation and the third one is guided by the body frame. Fig. 9 shows the mobile robot frame layout.

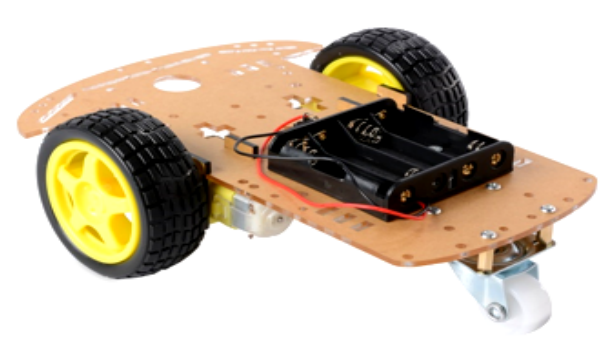

Figure 9. Two-wheel mobile robot structure

The overall structure of the mobile cell phone detection robot is shown in Fig. 10 (a) and (b). The robot includes the main components of cell phone detection circuit, NodeMCU, motor driver, jammer module and mini Wi-Fi camera. 


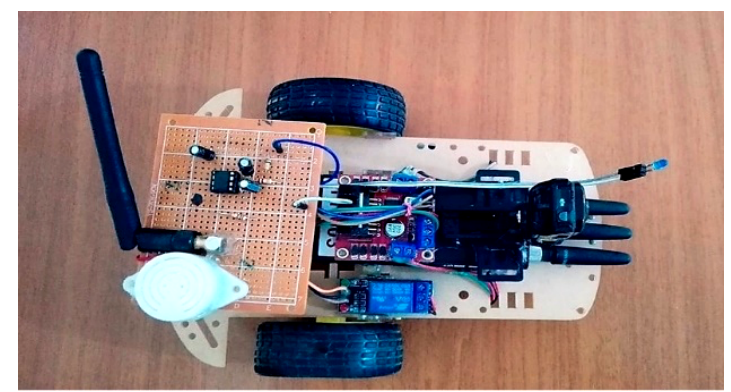

(a)

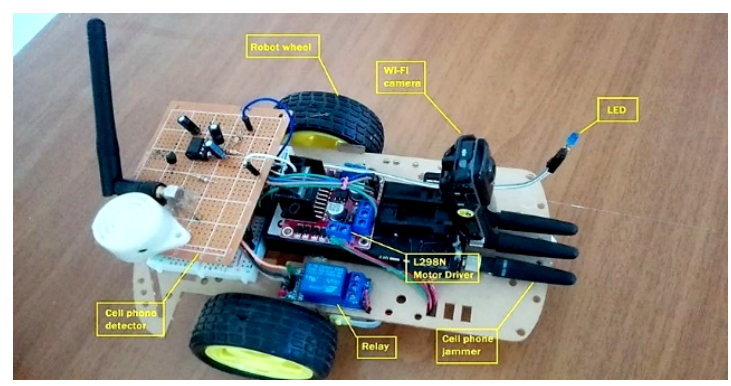

(b)

Figure 10. The overall mobile cell phone detection robot (a): top view (b) side labeled-parts view

\section{RESULTS AND DISCUSSION}

The mobile cell phone detection robot has been tested in an examination hall in which numerous of cell phones connections are active. In this hall, the active mobile handsets are set and distributed in lines to simulate real examinational environment. The mobile detection robot, which is being navigated by the main computer user, starts detecting the unauthorized using of phones. Once a detection is addressed, an alarm is consequently launched in form of buzzes and LED flashes. In addition, the main computer user receives detection signals, via the NodeMCU, in form of voltage measurements as an unauthorized access indication. Furthermore, the LED is intentionally placed in front of the camera on the robot so that the main computer user can notice the flashes whenever a malpractice is identified. Next, the main computer user stops the robot and directly fires on the jammer to distort that active RF signal.

The detection phase depends upon voltage levels measured from the operational amplifier LM358AD output in the detection circuit. It has been noticed that the voltage range when there are no active calls is $0.8-0.95$ volt, and that is the steady state mode. Once the voltage measurement level records more than that threshold range, the circuit status changes to the detection mode and the jammer is switched on to block any undesired signals by gradually raising their frequencies. Because of that progressive increase, the signal becomes less sensible to detection which leads to the steady state mode. Consequently, the signal is distorted and LED flashing is stopped so that the main computer user may switch off the jammer. It has been experimented that the jammer takes a couple of seconds to distort signals and approximately two minutes to drop the voltage down and going to the steady state mode. TABLE I shows voltage measurements for different distances. The robot can circularly detect signals within a diameter of (1.2 $\mathrm{m})$, and that is reasonably sufficient for the robot roaming over sitting rows of examination halls. The highest voltage has been 
recorded to be $(2.21 \mathrm{v})$ at the robot distant from a phone handset by $(20 \mathrm{~cm})$. In contrast, the lowest voltage of $(1.1 \mathrm{v})$ is measured with a phone that is $(60 \mathrm{~cm})$ distant. That means, the closer unauthorized phone is, the higher voltage level is.

TABLE I

MEASUREMENTS OF ACTIVE CALLS

\begin{tabular}{|c|c|c|}
\hline \multirow{2}{*}{$\begin{array}{c}\text { Distances } \\
\text { (Centimeters) }\end{array}$} & \multicolumn{2}{|c|}{ Measurements (Volts) } \\
\cline { 2 - 3 } & Detection mode & $\begin{array}{c}\text { Steady state mode (no } \\
\text { calls or jammer on) }\end{array}$ \\
\hline 20 & 2.21 & 0.8 to 0.95 \\
\hline 40 & 1.5 & 0.8 to 0.95 \\
\hline 60 & 1.1 & 0.8 to 0.95 \\
\hline
\end{tabular}

\section{CONCLUSION}

The detection and distortion of undesirable cellular RF signals have been presented in this work. That is accomplished by implementing a modified cell phone detection circuit, NodeMCU and a jammer module all instilled in a mobile robot. The robot driving and jammer switching are wirelessly controlled by a main computer via a specially designed HTML script in form of GUI control panel. The developed part of this work is the embedded system NodeMCU. Where it receives wireless commands from GUI in the main computer and sends them to the robot drivers and jammer by an interfacing circuit. The robot has been applied roaming in an examination hall and perfect detection and jamming of undesired signals have been achieved with different robot-to-phone distances. In addition to examination halls, this work is applicable in meeting rooms, private conferences or any areas in which the using of mobile phones is extremely constrained.

\section{REFERENCES}

[1] M. Kumar, N. Kaushal, H. Bhute, and M. K.Sharma, "Design of Cell Phone Operated Robot Using DTMF for Object Research," IEEE International Conference on Wireless and Optical Communications Networks (WOCN), Bhopal, India, July 2013.

[2] J. A. Dar, "Centralized Mobile Detection in Examination Hall Using Arduino Duemilanove (ATmega328)," International Journal of Scientific \& Engineering Research. India, Vol. 5, Issue 8, pp. 191-204, August 2014.

[3] A. S. Nyamawe and N. Mtonyole, "The Use of Mobile Phones in University Exams Cheating: Proposed Solution," International Journal of Engineering Trends and Technology, Vol.17, No.1, pp. 14-17, Nov 2014.

[4] T. Deshpande and N. Jahav "Active Cell Phone Detection and Display Using Atmega-8 Microcontroller," International Journal of Research in Engineering \& Technology, India, Vol. 3, Issue 8, pp. ,21-24, Aug 2015.

[5] R. Kumar, "Design and Implementation of Cell Phone Operated Metal Detector," International Journal of Research in Engineering, Technology and Science, India, Vol. VI, pp.1-9, July 2016.

[6] N. Gayathri and T. Sivasakthi "Presence of Active Mobile Phones and Hidden Camera Detection," International Journal of Computing Communication and Information System, India, Vol.8, No.1, pp. 01-07, 2016.

[7] E. Ataro, D. S. Madara and S. Sitat "Design and Testing of Mobile-Phone-Detector," Innovative Systems Design and Engineering, Vol.7, No.9, pp. 6-14, 2016.

[8] P. Ambulge, S. Bhosale, A. Mane and S.R. Sot "Metal Detector Robot Vehicle Operated by Android Application," International Journal of Innovative Research in Computer and Communication Engineering, Vol. 5, Issue 3, pp. 5887- 5890, March 2017.

[9] K. Parvateesam and G.A. ArunKumar "Presence of Active Mobile Phones and Hidden Camera Detection," International Journal of Science, Engineering and Technology Research, Vol. 6, Issue 4, pp. 689 -693, April 2017.

[10] Handson Technology, "User Manual V1.2 ESP8266 NodeMCU WiFi Devkit". 